\title{
Deformidad de Sprengel
}

\author{
D. Sanz Álvarez ${ }^{\mathrm{a}}$, MJ. Manuel Enguídanos ${ }^{\mathrm{b}}$ \\ 'MIR-Pediatría, Hospital Universitario 12 de Octubre. Madrid. España. \\ ${ }^{b}$ Pediatra. CS Los Rosales. Madrid. España.
}

\author{
Rev Pediatr Aten Primaria. 2009; I 1:63 I-8 \\ Débora Sanz Álvarez, debora_sanz@hotmail.com
}

\section{Resumen}

La deformidad de Sprengel se caracteriza por la elevación congénita de la escápula. Es la malformación de la cintura escapular más común. Se produce por la interrupción en el normal descenso de la escápula durante el período embrionario, de tal manera que ésta permanece en una posición elevada con respecto al tórax y al cuello.

Presentamos un caso clínico de un niño de 4 meses de edad al que, en su revisión del Programa de Salud Infantil, a la edad de 1 mes, se le detecta una deformidad de Sprengel en el lado izquierdo y se le asocian otras malformaciones, como escoliosis congénita, alteraciones vertebrales, torácicas y renales.

Palabras clave: Malformación escapular, Escoliosis.

Abstract

Sprengel's deformity is characterized by congenital elevation of the scapula. It is the most common congenital malformation of the shoulder girdle. Congenital elevation of the scapula is caused by an interruption in the normal embryonic caudal migration of the scapula, so that it rests in a high position in relation to the thorax and neck.

We report the case of 4 month infant, in whom Sprengel's deformity in the left side was detected in the 1 month well child review. It was associated with other malformations such as congenital scoliosis, renal, thoracic and vertebral abnormalities.

Key words: Scapula abnormalities, Scoliosis.

\section{Introducción}

La deformidad de Sprengel se caracteriza por la presencia de una elevación congénita de la escápula. Es una malformación compleja, que suele asociar displasia de la escápula y atrofia o hipopla- sia muscular. Es poco frecuente; aun así, es la malformación de la cintura escapular más común.

Se produce por una alteración en el normal descenso de la escápula, de tal manera que ésta permanece en una po-

Las autoras declaran no presentar conflictos de intereses en relación con la preparación y publicación de este artículo. 
sición elevada con respecto al tórax y al cuello. Puede ser una patología leve; logra incluso pasar desapercibida cuando no hay afectación estética ni funcional del hombro. Puede presentar diferentes niveles de gravedad según se dañen estas funciones o si se acompaña de otras malformaciones.

Presentamos un caso clínico observado en nuestra consulta y que está siendo controlado en el hospital de referencia.

\section{Caso clínico}

Acude a la revisión del Programa de Salud Infantil un lactante de 1 mes de vida. Antecedentes personales: en la ecografía fetal del tercer trimestre presentaba dilatación pielocalicial bilateral (derecha: 4,5 mm; izquierda: 4,1 mm). El parto fue por cesárea en la semana 41+4 de gestación por fallo de inducción. Su Apgar fue de 5/9 y precisó reanimación tipo III; permaneció en transición menos de 24 horas. Antecedentes familiares de interés: prima hermana paterna con escoliosis congénita. Exploración: Peso: 3,960 kg (percentil 25); talla: $53 \mathrm{~cm}$ (percentil 50); y perímetro cefálico: $37 \mathrm{~cm}$ (percentil 25). Se aprecian un buen estado general, cráneo con fontanela anterior de $1 \times 1 \mathrm{~cm}$, plagiocefalia leve, cuello corto con desvia- ción lateral de la cabeza al lado izquierdo y ligera limitación de la movilidad, así como escoliosis congénita cervical. Se observa una asimetría en la parte superior del tórax posterior, con elevación de la escápula izquierda: en la inspección se aprecia un hoyuelo cutáneo en esa escápula, la cual presenta un tamaño inferior y parece más horizontalizada con respecto a la contralateral. El resto de la exploración física y neurológica fue normal.

Ante los hallazgos de escápula elevada, escoliosis, tortícolis y plagiocefalia se decide su derivación al servicio de traumatología del hospital de referencia. Debido a sus antecedentes de dilatación pielocalicial se solicita una ecografía abdominorrenal de control.

En la revisión del cuarto mes la asimetría escapular es más manifiesta, se observa una mayor discrepancia entre el tamaño de ambas escápulas (la derecha mide $7 \mathrm{~cm}$, frente a los $4,5 \mathrm{~cm}$ de la izquierda), y la altura del ángulo inferior de ambas dista 2,5 cm. La movilidad del brazo está conservada, aunque existe una limitación de la abducción del hombro izquierdo (activa: $85^{\circ}$; pasiva: $115^{\circ}$ ). La plagiocefalia ahora es moderada, por lo que se decide derivar al servicio de neurocirugía para valorar ortesis craneal. 
Figura 1. Radiografía anteroposterior de tórax donde se aprecia la elevación de la escápula izquierda junto con otras alteraciones esqueléticas (vertebrales y torácicas).

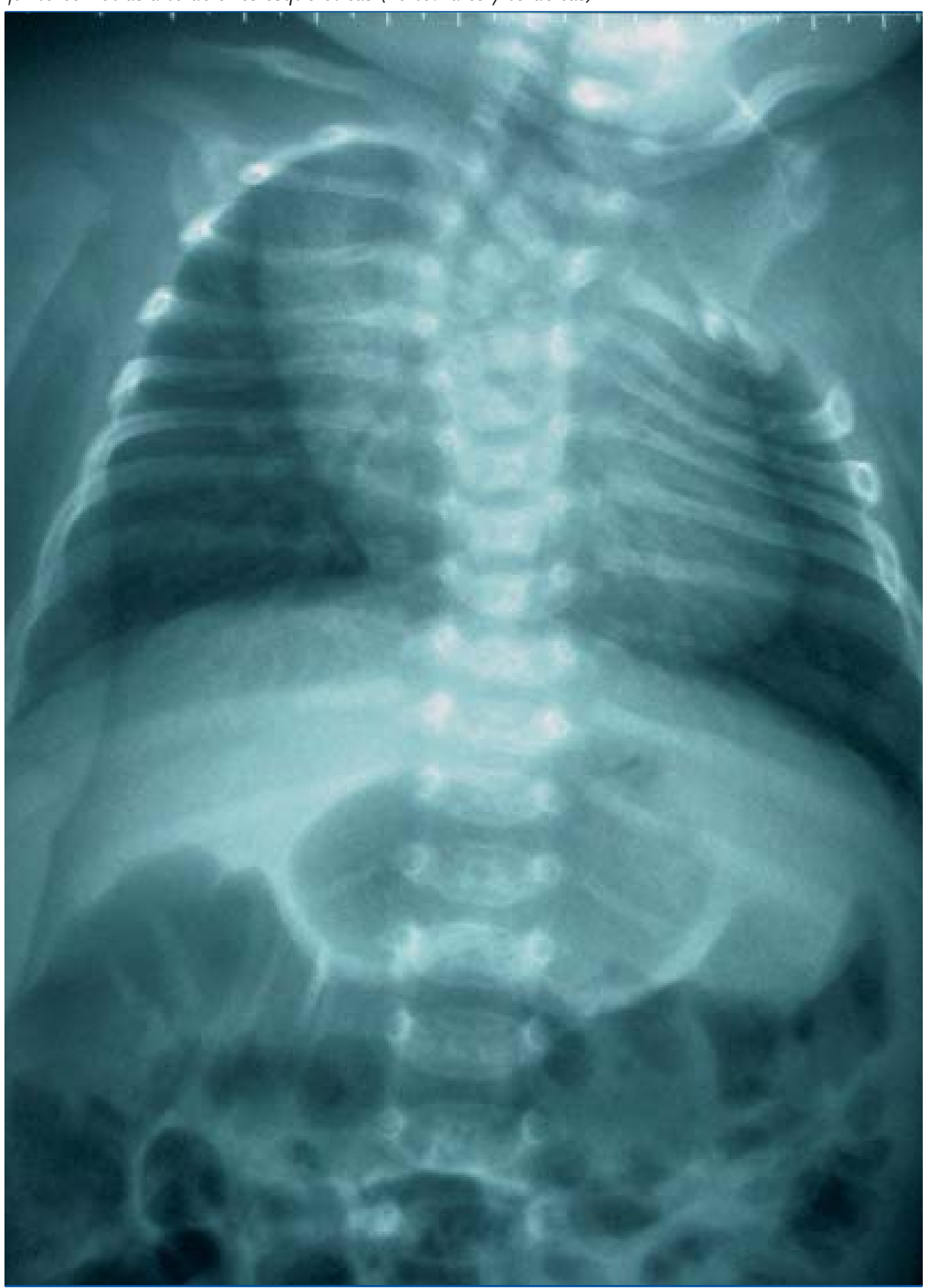

633

Revista Pediatría de Atención Primaria

Volumen XI. Número 44. Octubre/diciembre 2009 
Con el fin de confirmar el diagnóstico y descartar otras alteraciones asociadas se le realizan las siguientes pruebas complementarias:

- Ecografía renal (mayo de 2009): se aprecian una dilatación pielocalicial bilateral de grado II/IV, pelvis renal derecha de $9 \mathrm{~mm}$ e izquierda de $1 \mathrm{~cm}$.

- Ecografía de caderas (mayo de 2009) por sospecha de displasia de caderas: sin hallazgos significativos.

- Radiografía de columna completa y escápula (mayo de 2009): se observan alteraciones en la fusión de los cuerpos vertebrales de la columna dorsal alta y la cervical baja. Primera costilla izquierda hipoplásica. Costillas 2. ${ }^{\mathrm{a}}$ a $5 .{ }^{\mathrm{a}}$ izquierdas con disminución del espacio intercostal. Elevación de escápula izquierda ("escápula alada").

- Radiografía de columna lateral (junio de 2009): se objetivan alteraciones de segmentación de los cuerpos vertebrales de la columna vertebral dorsal alta y cervical baja, así como elevación de la escápula izquierda.

- Ecocardiograma y electrocardiograma: normales.

\section{Discusión}

En 1863 Eulenberg describió el primer caso de elevación congénita de la escápula, aunque sería Sprengel, en 1891, quien daría nombre a esta deformidad tras la descripción de cuatro casos. Esta malformación se caracteriza por una escápula alta con respecto al tórax y el cuello. En el $25-30 \%$ de los casos puede hallarse el hueso omovertebrall-3, que consiste en una estructura congénita anómala, formada por tejido fibroso, cartílago, hueso o ambos, que conecta el ángulo superior de la escápula con la columna vertebral. Este hueso no se encuentra en nuestro paciente, lo cual es beneficioso, puesto que su presencia conlleva una mayor gravedad del cuadro.

La prevalencia de esta patología no está documentada, pero como pertenece al conjunto de las denominadas enfermedades raras (declarada así por el Ministerio de Sanidad), tiene que ser por definición menor de 1 caso por cada 2.000 personas. La proporción hombre:mujer es de 1:3 $3^{1,4}$. Se ve afectada con mayor frecuencia la escápula izquierda, como ocurre en nuestro caso ${ }^{1,2,4}$.

La etiología es desconocida. Se han implicado factores genéticos. Generalmente es una patología esporádica, aunque se han descrito casos de herencia autosómica dominante ${ }^{5}$. Dentro de 
la patogenia de la deformidad de Sprengel es importante conocer la embriología del desarrollo de la escápula; ésta tiene su origen en un apéndice cervical que normalmente se diferencia a la altura de la $4 .^{a}-5{ }^{a}-6 .{ }^{a}$ vértebra cervical, en la quinta semana de gestación. Esta estructura normalmente desciende al tórax al final del tercer mes de vida intrauterina. Cualquier impedimento en su descenso da lugar a una escápula hipoplásica y elevada6.

La deformidad de Sprengel puede clasificarse clínicamente según su gravedad basándose en los grados de Cavendish": grado 1 (la deformidad es muy suave, los hombros están casi al mismo nivel y la deformidad no se aprecia con la ropa); grado 2 (la deformidad es leve, los hombros se sitúan casi al mismo nivel, pero la parte superomedial de la escápula elevada se aprecia como un bulto); grado 3 (la deformidad es moderada, es visible y el hombro afecto se eleva $2-5 \mathrm{~cm}$ con respecto al opuesto); grado 4 (la deformidad es grave, la escápula está muy alta, con el ángulo superomedial en el occipucio, con pterygium colli y brevicollis). El caso que nos ocupa puede englobarse dentro del grado 3 de Cavendish, ya que

Figura 2. Radiografía anteroposterior ampliada donde se visualiza la elevación escapular izquierda.

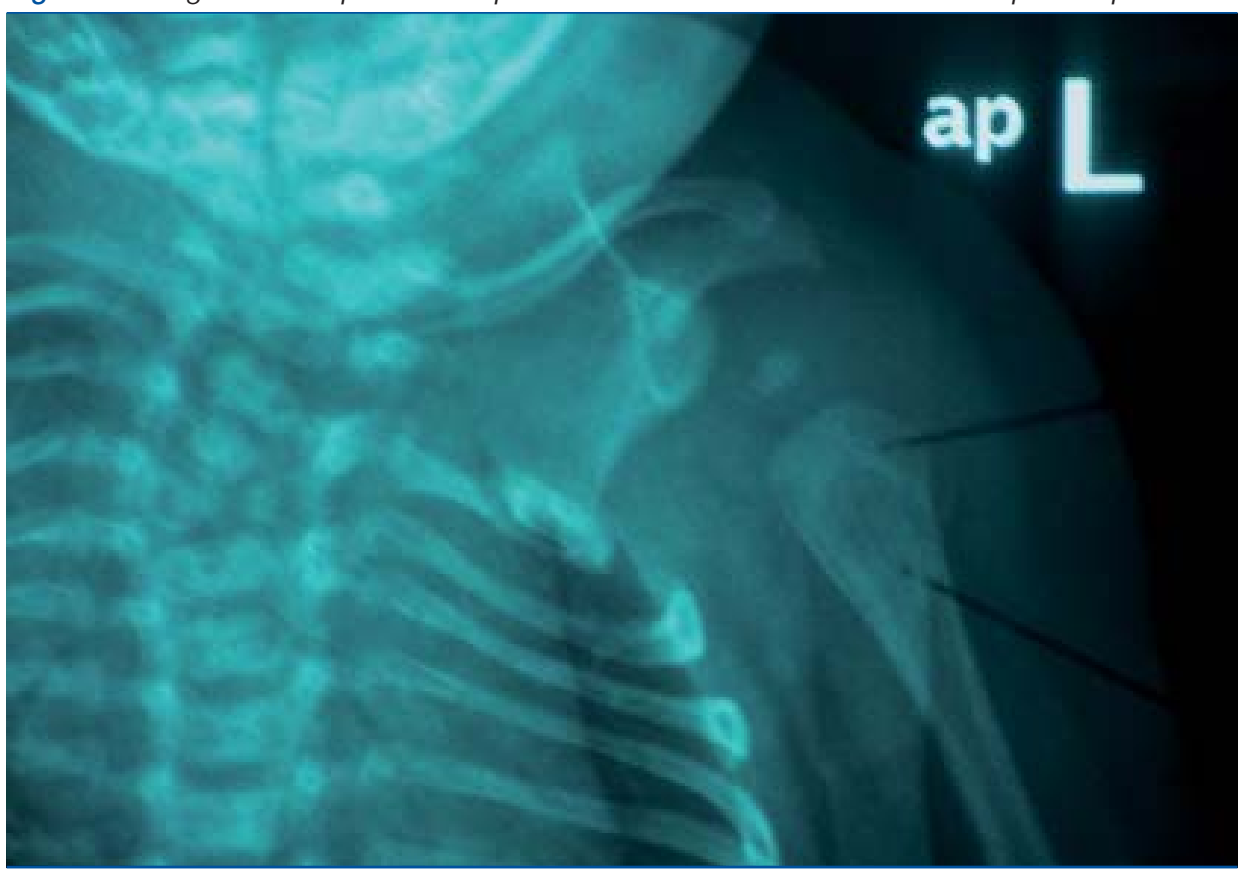


en la última exploración realizada en julio de 2009 presentaba una diferencia de altura entre ambas escápulas de 2,5 cm. Son importantes la observación y la evaluación continuas de estos pacientes, puesto que la apariencia estética suele deteriorarse en períodos de crecimiento rápido del cuello ${ }^{1}$, como sucede en los primeros años de vida.

Para el diagnóstico de la deformidad de Sprengel, en un paciente con sospecha de ella, los primeros estudios que deben realizarse son una radiografía de tórax anteroposterior, que nos permite valorar el desplazamiento de la escápula y medirlo con el método de Leibovic? así como una radiografía lateral, que facilita la visualización de anomalías de la columna vertebral que pueden asociarse y del hueso omovertebral si está presente. La realización de una tomografía computarizada se supedita a obtener una mejor visualización de la anatomía de la zona, con el fin de planificar la intervención quirúrgica o bien corroborar la existencia y la morfología del hueso omovertebral. Nuestro paciente sólo ha sido sometido a estudios radiográficos simples por el momento, porque la información que éstos han aportado es suficiente para descartar patologías de gravedad, así como por su corta edad.
La deformidad de Sprengel, además de presentar alteraciones en la posición de la escápula, y en muchos casos en la conformación de dicho hueso, puede asociar trastornos en los músculos regionales (trapecio, romboidal, elevador de la escápula), que pueden manifestarse como ausencia del músculo, hipoplasia o fibrosis, lo que provoca limitación del movimiento de la articulación del hombro, trastornos en la parrilla costal (ausencia o hipoplasia costal, costillas fusionadas, asimetrías, costilla cervical), trastornos en la columna vertebral y médula espinal -escoliosis congénita (es más frecuente cervicotorácica o torácica superior), síndrome de Klippel-Feil, espina bífida y diastematomielia- ${ }^{1,4,7}$; incluso se ha encontrado asociación con paladar hendido ${ }^{5,8}$. También se han descrito casos en los que se asociaban alteraciones en otros órganos, como a nivel renal (dilatación pielocalicial, agenesia renal, riñón ectópico) $)^{4,7-9}$ y cardíaco (situs inversus, cardiopatía congénita) ${ }^{2,4}$.

Debido, pues, a la posibilidad de asociarse con patologías de gravedad, es preciso, en todo paciente en el que se diagnostique una deformidad de Sprengel, hacer un estudio con el fin de descartar otras entidades que, a simple vista, no sean tan manifiestas como la elevación de la escápula. Los estudios 
más prioritarios son los de columna vertebral y tórax, ya que son las localizaciones más frecuentemente alteradas debido a su proximidad estructural.

En nuestro paciente, la exploración física y las pruebas realizadas han permitido detectar las siguientes anomalías asociadas: dilatación pielocalicial bilateral de grado II/IV, escoliosis cervical congénita derecha, alteraciones en la segmentación de las vértebras cervicales bajas y dorsales altas, hipoplasia de la 1. a costilla izquierda, disminución del espacio intercostal de la $2 .^{a}-5 .{ }^{a}$ costilla izquierda y plagiocefalia moderada.

El tratamiento de la deformidad de Sprengel se compone de dos partes: el tratamiento médico que se basa en la terapia física, en la que se usan los ejercicios para mantener el rango de movilidad individual y la fuerza de los músculos periescapulares y el tratamiento quirúrgico, indicado a partir del grado 2 de Cavendish, en pacientes con gran afectación estética y/o funcional. Se basa en la recolocación de la escápula y/o en la escisión de la parte superomedial de ella y del hueso omovertebral si está presente ${ }^{7,910}$. La edad óptima para la intervención es un tema controvertido; sin embargo, la mayoría de los autores recomienda que la cirugía se lleve a cabo antes de los 8 años con el fin de obtener mejores resultados, ya que a esta edad la anatomía se halla suficientemente desarrollada y posee un estado de plasticidad biológica que le permite acomodarse a los cambios inducidos por la operación $n^{4}$. Las técnicas más utilizadas en la corrección de la deformidad de Sprengel son la de Green modificada (1990)7 la de Woodward $(1961)^{10}$ y la Mears (2001)9. Aquellas técnicas que incluyen resección de la parte superior de la escápula obtienen mejores resultados ${ }^{3}$. Toda cirugía tiene un riesgo. Dentro de las complicaciones más frecuentes se encuentran la escápula alada, que puede ser el resultado de la reinserción incompleta del músculo serrato anterior; la lesión del plexo braquial, la más grave; y la formación de cicatrices queloides.

La trascendencia de este caso radica en su baja frecuencia y en la necesidad de detectarlo en edades tempranas, lo cual suele estar favorecido por su gravedad o por la presencia de trastornos asociados ${ }^{3}$. Asimismo, es preciso resaltar la importancia de los controles de salud en el Programa de Salud Infantil en Atención Primaria, ya que en nuestro caso nos permitió detectar la deformidad de manera precoz e iniciar la derivación a servicios especializados de forma temprana, asegurando de esta manera un control y un seguimiento adecuados a la patología del niño. 


\section{Bibliografía}

1. Cavendish ME. Congenital elevation of the scapula. J Bone Joint Surg Br. 1972;54:395-408.

2. Carson WG, Lovell WW, Whitesides TE. Congenital elevation of the scapula: surgical correction by the Woodward procedure. J Bone Joint Surg Am. 1981;63:1199-207.

3. Farsetti $P$, Weinstein $S L$, Caterini $R$, De Maio F, Ippolito E. Sprengel's deformity: Longterm follow-up study of 22 cases. J Pediatr Orthop B. 2003;12:202-10.

4. Grogan DP, Stanley EA, Bobechko WP. The congenital undescended scapula. Surgical correction by the woodward procedure. J Bone and Joint Surg Br. 1983;65:598-605.

5. Hodgson SV, Chiu DC. Dominant transmission of Sprengel's shoulder and cleft palate. J Med Genet. 1981;18:263-5.
6. Matsuoka T, Ahlberg PE, Kessaris N, lannarelli $P$, Dennehy $U$, Richardson WD, et al. Neural crest origins of the neck and shoulder. Nature. 2005;436:347-55.

7. Leibovic SJ, Ehrlich MG, Zaleske DJ. Sprengel deformity. J Bone Joint Surg Am. 1990;72: 192-7.

8. Mooney JF 3rd, White DR, Glazier S. Previously unreported structure associated with Sprengel deformity. J Pediatr Orthop. 2009;29: 26-8.

9. Mears DC. Partial resection of the scapula and a release of triceps for management of Sprengel's deformity. J Pediatr Orthop. 2001;21: 242-5.

10. Woodward JW. Congenital elevation of the scapula. Correction by release and transplantation of muscle origins: a preliminary report. J Bone Joint Surg Am. 1961;43:219-28. 